Ann. Geophysicae 17, 43-52 (1999) @ EGS - Springer-Verlag 1999

\title{
The relationship between electric fields, conductances and currents in the high-latitude ionosphere: a statistical study using EISCAT data
}

\author{
J. A. Davies and M. Lester \\ LE1 7RH, UK \\ Received: 26 February 1998 / Revised: 4 June 1998 / Accepted: 8 June 1998
}

Radio and Space Plasma Physics Group, Department of Physics and Astronomy, University of Leicester, University Road, Leicester,

\begin{abstract}
The relationship between electric fields, height-integrated conductivities and electric currents in the high-latitude nightside electrojet region is known to be complex. The tristatic nature of the EISCAT UHF radar facility provides an excellent means of exploring this interrelationship as it enables simultaneous estimates to be made of the full electric field vector and the ionospheric Hall and Pedersen conductances, further allowing the determination of both field-perpendicular electric current components. Over $1300 \mathrm{~h}$ of common programme observations by the UHF radar system provide the basis of a statistical study of electric fields, conductances and currents in the high-latitude ionosphere, from which preliminary results are presented. Times at which there is significant solar contribution to the ionospheric conductances have been excluded by limiting the observations according to solar zenith angle. Initial results indicate that, in general, the times of peak conductance, identified from the entire set of EISCAT observations, do not correspond to the times of the largest electric field values; the relative contribution of ionospheric conductance and electric field to the electrojet currents therefore depends critically on local time, a conclusion which corroborates work by previous authors. Simultaneous measurements confirm a tendency for a decrease in both Hall and Pedersen conductances to be accompanied by an increase in the electric field, at least for moderate and large electric field value, a tendency which is also identified to some extent in the ratio of the conductances, which acts as an indicator of the energy of precipitating particles.
\end{abstract}

Key words. Ionosphere (auroral ionosphere; electric fields and currents).

Correspondence to: J. A. Davies

\section{Introduction}

Electric currents in the lower ionosphere, a manifestation of magnetosphere-ionosphere coupling, can be intensified through enhancements in the conductivity, enhancements in the electric field, or some combination of both. The relative importance of electric field and conductivity enhancements in the intensification of the electrojet currents varies significantly. The conductivity and electric field are, however, not totally independent, in the sense that spatial non-uniformity in the conductivity results in changes in the electric field. Previous authors have suggested that the eastward electrojet is a signature of magnetospheric convection whereas the westward electrojet, at least in the midnight sector, represents current intensification during substorms (e.g. Baumjohann, 1983). Radar observations support this view in that, while the eastward electrojet is controlled by northward electric fields, the westward electrojet current appears to be dominated by the Hall conductivity associated with precipitating auroral electrons (e.g. Brekke et al., 1974; Horwitz et al., 1978). Later work, documented by Kamide and Vickrey (1983) and reviewed by Kamide and Kokubun (1996), further delineates the westward electrojet into two distinct regions, the conductivity dominated region and, at later local times, a region of current controlled by southward electric fields. The location of this transition within the westward electrojet, between the region of current driven predominantly by enhanced conductivity and the electric field dominated region, like the transition between the eastward and westward electrojets themselves, depends on latitude, occurring at earlier local times at higher latitudes (e.g. Senior et al., 1982; Kamide and Vickrey, 1983; Foster et al., 1986). In other words, the poleward half of the morning side auroral electrojet is dominated by the electric field, while its equatorward portion is dominated by high conductivity (Kamide and Kokubun, 1996). Kamide and Kokubun (1996) coin the 
phrase the 'two-component auroral electrojet' to describe currents over the entire polar region in terms of, firstly, a system related to the twin-cell convection pattern and secondly, currents within the westward electrojet in the dark sector in turn related to the threedimensional current wedge system. Kamide and Nakamura (1996) point out that it is likely that, in auroral electrojet activity, the former provides a voltage source and the latter, a current source resulting from disruption of the cross tail current. Thus, which of the two processes dominates in the overall electrojet structure will depend critically on substorm phase, with the conductivity-rich electrojet in the midnight sector dominant at the expansion phase of substorms and the electric field-rich electrojet the main contributor during the growth and recovery phases (Kamide and Kokubun, 1996).

Ionospheric currents have mainly been studied from the point of view of the signatures which they produce in the ground magnetometer traces, although this requires, as input, profiles of the conductivity, usually from models (e.g. Kamide and Richmond, 1982). A less widely used method for studying the currents which flow in the ionosphere uses incoherent scatter radar observations from which estimates of the electric field and height profiles of both the Hall and Pedersen conductivity may be made (e.g. Kamide and Vickrey, 1983; Brekke et al., 1990; Kirkwood et al., 1988; Aikio and Kaila, 1996; Lester et al., 1996, amongst others). The majority of these studies consider only specific intervals whereas in the present study more than $1300 \mathrm{~h}$ of EISCAT observations, spanning the years 1992 to early 1997, provide the basis for a statistical analysis of conductances, electric fields, ionospheric currents and their interrelationship.

\section{Experimental arrangement}

The following investigation is based on observations taken by the EISCAT UHF radar in version $\mathrm{K}$ of common programme 1 (CP-1-K) mode of operation and version $\mathrm{E}$ of common programme 2 (CP-2-E). In the EISCAT CP-1 mode, the beam from the UHF transmitter is aligned along the local F-region magnetic field direction which, for a radar located at Tromsø, is at an elevation of around $77^{\circ}$ and a geographic azimuth of $182^{\circ}$. In version $\mathrm{K}$ of the $\mathrm{CP}-1$ programme, the remote site radars, at Kiruna and Sodankylä, intersect the transmitter beam at a fixed F-region altitude, centred around $278 \mathrm{~km}$. In CP-2, the transmitter performs a four position scan, one position of which is field-aligned, with a dwell time for each position of the scan of some $90 \mathrm{~s}$, giving a total cycle time of $6 \mathrm{~min}$. The remote site radars follow the transmitter beam scan, providing tristatic observations at a single F-region altitude, $278 \mathrm{~km}$ for CP-2-E. Only observations from the fieldparallel pointing direction of CP-2-E are employed in the present study.

In $\mathrm{CP}-1-\mathrm{K}$ and $\mathrm{CP}-2-\mathrm{E}$, identical pulse schemes are transmitted. A transmitted pulse of length $350 \mu \mathrm{s}$ provides long pulse measurements, which extend over 21 range gates along the magnetic field direction between some 140 and $600 \mathrm{~km}$ altitude, with a gate separation, in altitude, of $22 \mathrm{~km}$. High altitude resolution observations in the E- and lower F-region are obtained by means of a 16 baud, strong condition, alternating code, with a baud length of $21 \mu \mathrm{s}$. In the field-parallel pointing direction, the 61 signal gates provided by the alternating code scheme extend from 86 to $270 \mathrm{~km}$ altitude, with a gate separation of $3.1 \mathrm{~km}$. In addition, both a $21 \mu \mathrm{s}$ and a $40 \mu$ s power profile are transmitted, the former of which provides the zero lag lacking in the alternating code autocorrelation function. Received signals are integrated over $5 \mathrm{~s}$.

CP-1-K observations from each site were post-integrated at a temporal resolution of $2 \mathrm{~min}$ and CP-2-E observations were post-integrated over the dwell time at each scan position. Standard incoherent scatter analysis was performed, providing estimates of ion and electron temperature, plasma density and ion velocity from both the alternating code and long pulse schemes; alternating code analysis does, however, assume an equality of the ion and electron temperatures at altitudes below $106 \mathrm{~km}$. In order to eliminate the least reliable data, a signal-to-noise threshold of $2 \%$ was applied to the entire data set. Observations are limited to those taken along the field-parallel pointing direction, as the authors are considering simultaneous ionospheric signatures at Eand F-region altitudes. No assumptions regarding the spatial scales of ionospheric features are, thus, necessary.

\section{Derivation of parameters}

The methods by which the ionospheric electric field, the Hall and Pedersen conductances and the ionospheric currents are derived from the EISCAT observations are discussed below.

\section{Electric field}

Above some $200 \mathrm{~km}$ altitude, where ion-neutral collisions are infrequent, the ionospheric plasma is constrained to move only along the direction of the geomagnetic field, $\mathbf{B}$, unless a perpendicular electric field, $\mathbf{E}$, is imposed, under which conditions the plasma travels, orthogonal to the magnetic field, with the $\mathbf{E} \times \mathbf{B}$ drift. The tristatic nature of the EISCAT UHF radar system enables the full vector velocity to be determined in the common volume of intersection of the three receiver beams; CP-1-K and CP-2-E provide continuous tristatic measurements at the F-region altitude of $278 \mathrm{~km}$. The ionospheric electric field can, therefore, be simply derived from the long pulse measurements of F-region perpendicular ion velocity, given an appropriate estimate of the magnetic field strength, in this case from the IGRF model of the geomagnetic field. 


\section{Conductances}

The Hall and Pedersen conductivities, $\sigma_{H}$ and $\sigma_{P}$ respectively, are derived using the classical expressions which, in the presence of several $(j)$ ion species, may be written in the form of Eq. (1a) and (1b) below (e.g. Brekke and Moen, 1993)

$\sigma_{\mathrm{H}}=\frac{\mathrm{N}_{\mathrm{e}} \mathrm{e}}{\mathrm{B}}\left(\frac{\omega_{\mathrm{e}}^{2}}{v_{\mathrm{en}}^{2}+\omega_{\mathrm{e}}^{2}}-\sum_{\mathrm{i} j} \mathrm{P}_{\mathrm{i} j} \frac{\omega_{\mathrm{i} j}^{2}}{v_{\mathrm{i} j \mathrm{n}}^{2}+\omega_{\mathrm{i} j}^{2}}\right)$

$\sigma_{\mathrm{P}}=\frac{\mathrm{N}_{\mathrm{e}} \mathrm{e}}{\mathrm{B}}\left(\frac{\omega_{\mathrm{e}} v_{\mathrm{en}}}{v_{\mathrm{en}}^{2}+\omega_{\mathrm{e}}^{2}}+\sum_{\mathrm{i} j} \mathrm{P}_{\mathrm{i} j} \frac{\omega_{\mathrm{i} j} v_{\mathrm{i} j \mathrm{n}}}{v_{\mathrm{i} j \mathrm{n}}^{2}+\omega_{\mathrm{i} j}^{2}}\right)$

in which $N_{e}$ represents the electron density, $e$ is the electronic charge, $B$ is the magnetic field strength and $\omega_{e}$ and $v_{e n}$ represent the electron gyrofrequency and electron-neutral collision frequency, respectively. $\omega_{i j}$ and $v_{i j n}$ are the gyrofrequency and collision frequencies for the jth ion, the proportion of which is given by $P_{i j}$.

The height-integrated Hall and Pedersen conductivities, the ionospheric conductances, denoted by $\Sigma_{H}$ and $\Sigma_{P}$ respectively, are integrated, in the present study, over the altitude range 86 to $200 \mathrm{~km}$ and incorporate alternating code measurements of electron density. Alternating code profiles provide good quality estimates of E- and lower F-region electron density, which are far better than those yielded by the multi-pulse schemes used previously. In the majority of previous studies, conductances have been derived from the raw power, i.e. the power profile, rather than a true electron density (e.g. Brekke and Hall, 1988; Schlegel, 1988; Aikio and Kaila; 1996). In contrast to the fitted electron densities from the alternating code scheme, the raw power is not corrected for any inequality in the ion and electron temperatures or Debye length effects (e.g. Brekke and Moen, 1993; Moen and Brekke, 1993). Brekke and Moen (1993), in agreement with Senior (1991), suggest that the difference between applying raw and true electron densities for conductance calculations can amount to anywhere up to $20 \%$, although the discrepancy will depend critically on the prevailing conditions.

The integration boundaries will also obviously influence the accuracy of the conductance estimates. Previous authors (e.g. Senior, 1991; Brekke and Hall, 1988; Schlegel; 1988; Lilensten et al., 1996) agree that in the daytime, the contribution from altitudes above $200 \mathrm{~km}$, the upper boundary adopted in this study, is at most a few percent in the case of the Pedersen conductance and negligible for the Hall conductance. On the nightside, it is the strength of any precipitation which will determine the upper integration boundary required to describe fully the conductances. For example, for a collimated electron beam of $5 \mathrm{keV}$, which would release its energy in the conductivity layer, an upper boundary of some $150 \mathrm{~km}$ would be adequate to include all contributions to the conductances. Hardy et al. (1987) state that precipitating electrons of energies less than $500 \mathrm{eV}$ will contribute very little to the conductivities since they produce ionisation at altitudes where the collision frequencies are extremely low. In this study, the lower altitude boundary of integration is determined by the lowest range of the alternating code scheme, around $86 \mathrm{~km}$ altitude. The contribution to the Pedersen conductance from below this altitude would be highly unlikely to be significant. The contribution to the Hall conductance could, however, be more substantial; Schlegel (1988) estimated that, in cases of extremely energetic precipitation, as much as $15 \%$ of the Hall conductance may originate from altitudes between 75 and $90 \mathrm{~km}$.

For both the Hall and Pedersen conductances, the ion term is evaluated as the sum over three ion species, $\mathrm{O}^{+}, \mathrm{O}_{2}^{+}$and $\mathrm{NO}^{+}$. The relative proportion of each ion is taken from the ion composition model used in the standard analysis of EISCAT data. Within the altitude range of relevance to the present work, EISCAT analysis assumes a pseudo-molecular ion with a relative mass of 30.5 AMU corresponding to a mixture of $75 \%$ $\mathrm{NO}^{+}$and $25 \% \mathrm{O}_{2}^{+}$, although results are relatively insensitive to the exact ratio of $\mathrm{NO}^{+}$to $\mathrm{O}_{2}^{+}$adopted, and an atomic population comprising solely of $\mathrm{O}^{+}$. The relative proportion of atomic oxygen ions and molecular ions varies, of course, with altitude. It should be noted that the ion composition model adopted in standard EISCAT analysis, and consequently in the present conductance calculations, is totally time invariant, a somewhat physically unrealistic situation, especially under active conditions where substantial modification of the ion composition can result in response to processes such as ion frictional heating (e.g. Davies et al., 1995).

In calculating the collision frequency for each of the three ion species, interactions are considered with the three most abundant neutral gases, $\mathrm{N}_{2}, \mathrm{O}_{2}$ and $\mathrm{O}$. The electron-neutral collision frequency includes contributions from interactions between electrons and the same neutral species. Collisions within the plasma itself are neglected. Expressions used for deriving the frequency of each ion-neutral collision are those quoted by Schunk and Nagy (1980), with the exception of that for $\mathrm{O}^{+}-\mathrm{O}$ interactions which is amended in the light of more recent work (see later); electron-neutral collision frequencies are derived via the formulations of Schunk and Nagy (1978). The ion and electron temperatures used to derive the collision frequencies are those measured by the radar, the neutral temperature is taken from the MSIS90 model thermosphere, as are the neutral densities. The precise values of the collision frequencies which should be used in aeronomical calculations are somewhat uncertain, particularly in the case of $\mathrm{O}^{+}-\mathrm{O}$ interactions as discussed, for example, by Burnside et al. (1987). It is now generally accepted that the early theoretical formulations underestimate the $\mathrm{O}^{+}-\mathrm{O}$ collision frequency, but the factor by which it is underestimated, dubbed the 'Burnside factor' by Salah (1993), is still the subject of considerable debate. In the present study, the expression for the $\mathrm{O}^{+}-\mathrm{O}$ collision frequency presented by Schunk and Nagy (1980) is multiplied by a coefficient of 1.2 in 
the light of results of a recent experimental study documented by Davis et al. (1995). This value for the Burnside factor is consistent with that derived theoretically by Pesnell et al. (1993). It should be noted at this juncture that since the major contributions to both conductances arise almost exclusively from altitudes below $150 \mathrm{~km}$ - where the ion population is assumed to be purely molecular, even doubling the $\mathrm{O}^{+}-\mathrm{O}$ collision frequency has minimal effect on the derived values. The influence of the ion-neutral collision frequency in deriving conductances has been studied by Brekke and Hall (1988) and Kirkwood et al. (1988). Brekke and Hall (1988) compared the altitude profiles of the Hall and Pedersen mobility terms evaluated using two different collision frequency models; version 1 represented an earlier approach adopted by Brekke et al. (1974) in which simplistic expressions were adopted for the collision frequencies and no correction for reduced mass was made, in version 2 each ion- and electron-neutral interaction was considered separately. The authors noted that in choosing the latter more complete model, a model similar to that adopted in the present study, the Hall and Pedersen conductance ratio and the Hall conductance itself were, on average, lower than those from the original work of Brekke et al. (1974). Kirkwood et al. (1988) reported that varying the ionneutral collision frequency by $30 \%$ resulted in changes of around $5 \%$ and $15 \%$ in the Pedersen and Hall conductances, respectively, whereas, the conductances are relatively insensitive to the electron-neutral collision frequency.

The gyrofrequencies, evaluated for the electrons and each ion species, incorporate IGRF estimates of the magnetic field strength.

It is necessary to reiterate some comments of Brekke and Moen (1993) regarding the derivation of conductances under disturbed conditions, highlighting points which are particularly relevant to the present study. The expressions for the conductivities quoted above are based on kinetic theory of gases in thermal equilibrium. In the auroral ionosphere, however, non-isotropic and turbulent behaviour are frequently observed. Moreover, under such conditions measurements provided by incoherent scatter, especially those of plasma temperatures, are somewhat compromised. The analysis of incoherent scatter spectra is limited under active conditions by such factors as the modification of the ion composition, divergence of the temperatures of the different ion species and the occurrence of non-Maxwellian line-ofsight ion velocity distributions. The effect of these limitations is discussed more fully by authors such as Davies et al. (1995).

An additional problem in calculating the conductivities under active conditions results from the use of an atmospheric model to yield the neutral densities. Local dissipation of heat in the auroral upper atmosphere, due to frictional and turbulent heating mechanisms, can have a significant effect on the neutral composition at a given altitude which is not fully described in atmospheric models; geomagnetic activity is characterised in MSIS90 only according to a global index.

\section{Current density}

The total northward and eastward height-integrated current densities, $J_{N}$ and $J_{E}$, respectively (subsequently referred to simply as current densities for convenience), are derived from appropriate combinations of the Hall and Pedersen conductances and the northward and eastward components of the perpendicular electric field such that

$J_{N}=-\sum_{P} E_{N}-\sum_{H} E_{E}$
$J_{E}=-\sum_{P} E_{E}+\sum_{H} E_{N}$

in which $E_{N}$ and $E_{E}$ represent the northward and eastward components of the electric field, respectively. Resultant current densities are those integrated over the range 86 to $200 \mathrm{~km}$ as this corresponds to the range over which the conductances are integrated; some underestimation of the currents will, therefore, inevitably arise although the extent to which the currents will be underestimated will vary through the data set.

These equations (Eq. 2a,b) will only yield approximate estimates of the current components, not only due to inaccuracies inherent in determining the conductances. Currents flowing at different altitudes are different not only by virtue of the different conductivities at each height; the effective electric field at any height depends upon the velocity of the neutral wind, itself a height dependant parameter. In applying the expressions, in Eq. $(2 a, b)$ components of the electric field from the Fregion are used, essentially disregarding neutral wind induced currents.

Notwithstanding comments made previously, it is still extremely worthwhile using radar measurements to infer conductances and currents in the high-latitude ionosphere. Any method to calculate parameters such as these, be it from radars or magnetometers, is associated with its own set of assumptions and sources of inaccuracy; these must be borne in mind when interpreting the results.

\section{Results and discussion}

Estimates of the electric field, the height-integrated Hall and Pedersen conductivities, and, from these, estimates of the current densities are made throughout each common programme run. For each, the IGRF and MSIS90 models are run, employing appropriate levels of solar activity and geomagnetic activity.

Figure 1 illustrates the diurnal variation of the Hall conductance (upper panel), the Pedersen conductance (middle panel) and the ratio of the Hall and Pedersen conductances (lower panel). The median and upper and lower quartiles of each parameter are presented, binned with respect to universal time with a bin width of $30 \mathrm{~min}$; dashed lines illustrate values derived from the whole data set, full lines indicate the median and quartiles of the conductances and their ratio under conditions where the solar zenith angle exceeds $105^{\circ}$. 


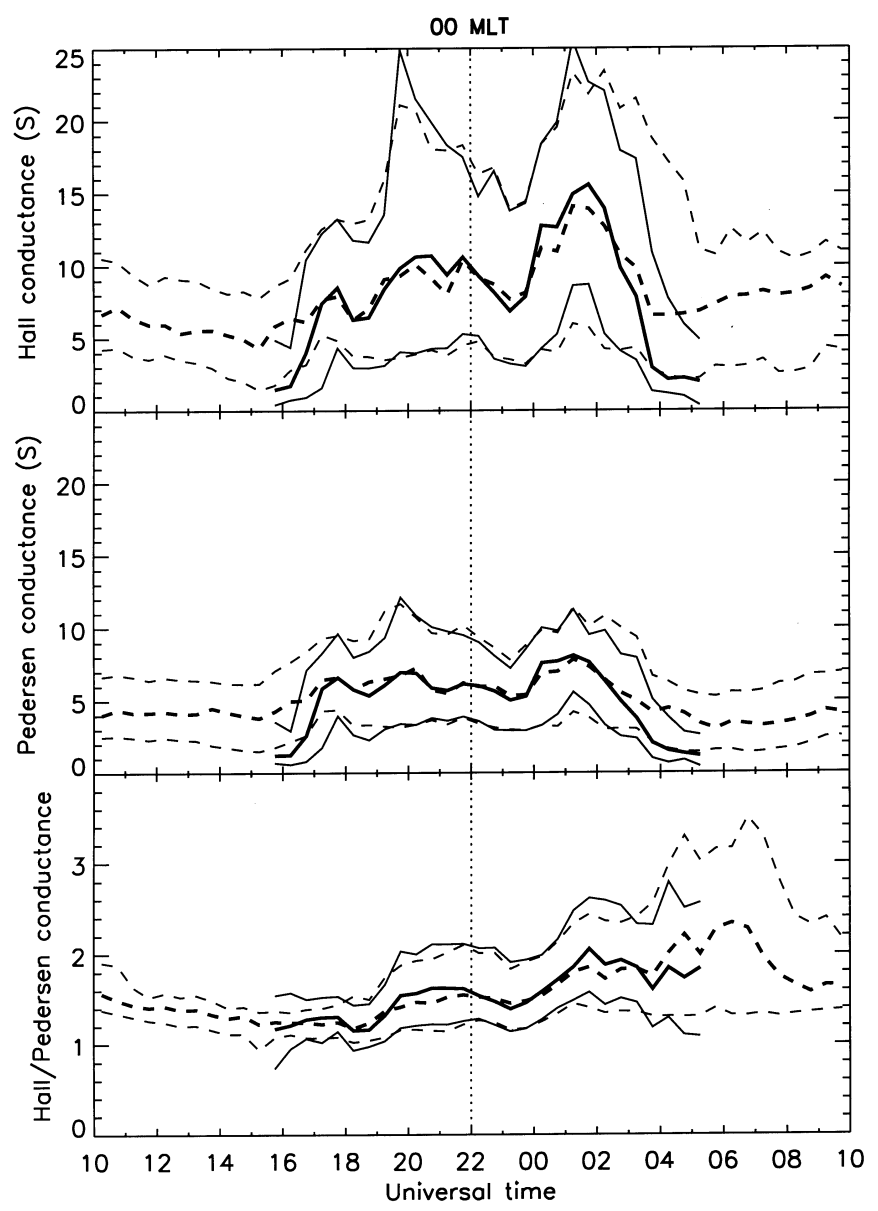

Fig. 1. UT variation of the Hall conductance (upper panel), the Pedersen conductance (middle panel) and the ratio of the Hall and Pedersen conductances (lower panel). The dashed lines indicate the median and upper and lower quartiles of each parameter, binned with respect to universal time with a bin width of $30 \mathrm{~min}$ and derived from the complete data set. The full lines are derived from data taken only when the solar zenith angle exceeds $105^{\circ}$. Plots are centred on midnight MLT, which corresponds to 22 UT (dotted line)

Plots are centred on midnight MLT, which corresponds to 22:00 UT (dotted line). The total number of observations in each half hour bin ranges from 400 to 500 for the entire data set. Restricting the observations in solar zenith angle reduces this number, preferentially of course on the dayside, and bins in which there are less than 30 observations are disregarded thus limiting the solid lines to prior to 06:00 UT and after 15:00 UT.

Limiting the observations in solar zenith angle, essentially to those taken under conditions of darkness, removes conductances which are produced, or influenced in part, by solar illumination. Whereas the dashed lines illustrate the diurnal variation of the total solar and particle-produced conductance at EISCAT, the solid lines are more indicative of the variation of conductances associated with auroral precipitation. The effect of introducing such a constraint appears negligible prior to 03:00 UT and after 17:00 UT.

Considering initially the full data set, both Hall and Pedersen conductances, of which the former exceeds the latter throughout the day, exhibit two distinct peaks.
These peaks, which arise on the nightside, are centred around 20:00 and 02:00 UT (roughly equivalent to 22:00 and 04:00 MLT, respectively). The post-midnight maximum in the Hall conductance exceeds that prior to midnight, with median values of around 14 and $10 \mathrm{~S}$ respectively, whereas, in the Pedersen conductance these peaks are more symmetric, with a median value of the order $7 \mathrm{~S}$. The nightside peaks are far more pronounced in the upper quartile, particularly in the Hall conductance, although the asymmetric behaviour of the Hall conductance is, if anything, less pronounced in the upper quartile. Interestingly, the pre-midnight peak in the upper quartile of the Pedersen conductance somewhat exceeds that in the post-midnight sector. There is, furthermore, some evidence of a third nightside peak, albeit small, in both conductances, centred around 18:00 UT. On the dayside, there is a clear increase in both conductances towards noon, the expected increase with increasing solar zenith angle, but the median conductances even at noon are significantly less than those on the nightside. Although previous authors (Hardy et al., 1987) have noted that there can be a significant contribution to the daytime conductances through precipitation, this is unlikely to be the case here as EISCAT almost invariably observes sub-aurorally on the dayside.

The ratio of the Hall and Pedersen conductances, $R$, provides information on the characteristic energy of the particle spectrum producing the enhanced ionisation on the nightside (e.g. Robinson et al., 1987), due to the particular sensitivity of the Hall conductance to this characteristic energy. A value for $R$ of unity indicates precipitating electrons with an average energy of the order of $2.5 \mathrm{keV}$, while $R$ of 2 indicates electron precipitation with mean energy $5.8 \mathrm{keV}$. In using the ratio of the Hall and Pedersen conductances to derive precipitating particle energy, it is assumed that the precipitation comprises solely of electrons which is not necessarily the case although Fuller-Rowell and Evans (1987) noted from their data that when the total energy fluxes become significant, it is relatively rare for the positive ions to contribute more than $20 \%$ of the total. Moreover, if the conductivities include a solar contribution, $R$ will not represent a true indicator of the hardness of precipitating electrons (e.g. Brekke et al., 1989).

The maximum in $R$ occurs around 06:00 UT, a time, however, when the conductances are low and are unlikely to be affected by particle precipitation. Two less distinct maxima in the ratio $R$ are evident, which coincide temporally with the pre- and post-midnight peaks in the conductances themselves. The pre-midnight peak in $R$, with a median value of 1.5 , is less than that in the post-midnight sector, of value around 2 . This would be anticipated from the fact that the post-midnight peak in the Hall conductance is greater than that during the pre-midnight hours, whereas the corresponding peaks in the Pedersen conductance are roughly symmetric. This evidence would suggest that, at least on a statistical basis, particle precipitation which occurs after midnight tends to be more energetic. It should be noted that the 
small peak evident in both the Hall and Pedersen conductances around 18:00 UT is also reflected in their ratio.

As noted earlier, if the data are constrained in solar zenith angle, to values exceeding $105^{\circ}$, little difference results within the interval 17:00 to 03:00 UT. Outside these times however, where the median and quartile values have been determined, they are considerably reduced, indicating that the high values are primarily solar-produced.

Several other authors have presented statistical distributions of ionospheric conductances from a variety of space and ground based data sources. Fuller-Rowell and Evans (1987), for example, present average global patterns of both Hall and Pedersen conductances for different levels of auroral activity. In this case, the conductances were derived from electron influx data monitored by a series of polar orbiting satellites, TIROS, NOAA-6 and NOAA-7. Similar, previous, work by Spiro et al. (1982) used data from two of the atmospheric explorer satellites, AE-C and AE-D. Global height-integrated conductivity patterns have also been inferred from ground magnetometer data (Ahn et al., 1983) and optical observations (Craven et al., 1984). Senior (1991) compared solar and particle contributions to auroral height-integrated conductivities using $900 \mathrm{~h}$ of EISCAT common programme 3 measurements taken between 1984 and 1987. The authors derived an empirical model of the solar-produced conductances from their entire data set which related the conductances to the solar zenith angle. The particle contribution was then inferred by subtracting the modelled solar contribution from the conductance observations; the authors further classified the particle-produced conductances according to geomagnetic activity. Previous related radar studies had mainly limited their analysis to quiet days, to isolate the solar-produced conductances (e.g. Vickrey et al., 1981; Schlegel, 1988; Brekke and Hall, 1988).

Particle-produced Hall and Pedersen conductances from the invariant latitude of $66^{\circ}$ (which is close to the location of the present observations) were compared by Senior (1991) to model results. The model used by Senior (1991) was that of Hardy et al. (1987), who derived a global empirical conductance model, for seven levels of geomagnetic activity as characterised by $K_{p}$, from electron precipitation characteristics measured on board the DMSP F2 and F4 satellites and P78-1 from the Satellite Test Program. The median values of conductance on the nightside from the present study are comparable to the mean particle-produced conductances derived by Senior (1991) at this latitude for average levels of geomagnetic activity. The doublepeaked form of nightside conductances clearly observed in this study are not, however, so obvious in results of Senior (1991), perhaps due in part to the far lower number of observations at this latitude available to Senior (1991), as the author used a scanning experiment to increase the latitudinal coverage of their study and further classified the conductances according to $K_{p}$. This feature is, however, more clearly reproduced by the model of Hardy et al. (1987). The twin peaks in both the modelled nightside Hall and Pedersen conductance, although evident for all levels of geomagnetic activity, are much more well defined during active conditions. This probably relates them more to the upper quartiles of Fig. 1, since the present conductance dataset indicates the well observed tendency for increasing conductances to be associated with increasing geomagnetic activity (e.g. Senior, 1991; Hardy et al., 1987; Fuller-Rowell and Evans, 1987), the latter is not presented illustratively here due to the scarcity of conductance estimates under more active conditions. Fuller-Rowell and Evans (1987) noted that for geomagnetically quiet conditions, the magnitude and distribution of the Hall and Pedersen conductances were virtually identical, a result which appears to be borne out in the present study in view of the comparable values of lower quartiles of both heightintegrated conductivities. It should also be pointed out that the third, small, peak observed in the conductances at around 18:00 UT is consistent with a similar feature which is evident in the model results of Hardy et al. (1987), although only for values of $K_{p}$ of 4 and above. Although the post-midnight peak in conductance revealed by the current work probably relates to a peak in precipitation of relatively hot electrons, those of average energy exceeding $600 \mathrm{eV}$ (Hardy et al., 1985), the results of Fuller-Rowell and Evans (1987) and Hardy et al. (1985) show that the time of the hardest electron precipitation, to which the Hall conductance is particularly sensitive, and that of maximum overall electron energy influx are highly variable.

A rigorous comparison with previous results is not attempted since the present work aims more to compare the relative importance of conductance and electric field as a mechanism for current generation in the highlatitude ionosphere.

The universal time variation of the electric field observed by EISCAT is illustrated in Fig. 2 where the upper panel represents the diurnal variation of the magnitude of the magnetic field-perpendicular electric field, the middle panel, its north-south component (positive northward), and the lower panel, the east-west component of the perpendicular electric field (positive eastward). The general layout of Fig. 2 is similar to that of Fig. 1. The number of observations in each bin is somewhat less than for the conductances (Fig. 1), since electric field estimation requires simultaneous velocity measurements from the three receiver sites, with the signal-to-noise ratio at each site exceeding $2 \%$. The perpendicular electric field magnitude exhibits a doublepeaked appearance. The pre-midnight peak, centred around 16:00 UT, corresponds to a maximum in the orthogonal north-south electric field component in the northward direction and a much less well-defined peak around 01:00 UT corresponds to the maximum in the southward electric field. The pre-midnight maximum has a median value exceeding $25 \mathrm{mV} \mathrm{m}^{-1}$ compared to less than $20 \mathrm{mV} \mathrm{m}^{-1}$ for the peak in the post magnetic midnight sector. The east-west electric field is generally much less than that in the north-south direction, except in the region of the north-south electric field reversal 




Fig. 2. UT variation of the magnitude of the field-perpendicular electric field (upper panel), its north-south component (middle panel; positive northwards) and its east-west component (lower panel; positive eastwards). The format is identical to that of Fig. 1

which does not coincide in time with the reversal in the east-west electric field. Such small east-west electric fields may, in fact, be projections of large fields oriented normal to the direction of the auroral oval locally, since the oval is not exactly aligned magnetic east-west except near midnight. Limiting the electric field observations in solar zenith angle, again neglecting bins with less than 30 observations, appears to have only a slight effect on the values except by reducing the range of times over which the median values can be calculated. However, imposing the zenith angle condition does eliminate observations associated with the largest values of the electric field, which occur between some 15:00 and 17:00 UT.

What is important to the present study, is the comparison of Figs. 1 and 2, especially with respect to the times of the maxima in both the conductances and the electric field. It is clear that the peaks in the Hall and Pedersen conductance do not coincide temporally with those in electric field, with the pre-midnight peak in electric field occurring before the first peak in the conductances and the post-midnight maximum in electric field falling between the two peaks in conductance. It is also significant that the post-midnight peak in electric field, whilst being smaller in value than the peak in the pre-midnight sector, corresponds to values of conductance which are far higher than those associated with the latter. Both of these features suggest that larger conductivities tend to be associated with smaller electric fields and vice versa. The ability of EISCAT to provide simultaneous measurements of conductance and electric field enables this to be investigated more fully later in the paper.

Comparison of Figs. 1 and 2 reveals that the relative contribution of the conductance and electric field in driving ionospheric currents must vary significantly with local time. The universal time variation of the calculated electric current components are illustrated in Fig. 3.

The upper panel of Fig. 3 illustrates the diurnal variation of the north-south current density (positive northwards). The lower panel illustrates the corresponding variation of the east-west current density (positive eastwards). East-west currents far exceed north-south currents, except near 20:00 UT where the east-west current reverses direction. The east-west electrojet current may be approximately described by the product of the Hall conductance and the north-south electric field. The pre-midnight peak in the eastward electrojet current, which occurs near 17:00 UT, appears to be roughly co-located with the peak in the northward electric field, at least at the latitude of EISCAT, rather than the Hall conductance. Indeed, throughout the eastward electrojet, the electric field appears to constitute the dominant factor determining the current density, with the conductance remaining low. The westward electrojet current is clearly more complex in nature, but in general the current within this region appears to be dominated more by the Hall conductance rather than the electric field. Certainly the peak in the westward current corresponds more to the post-midnight peak in

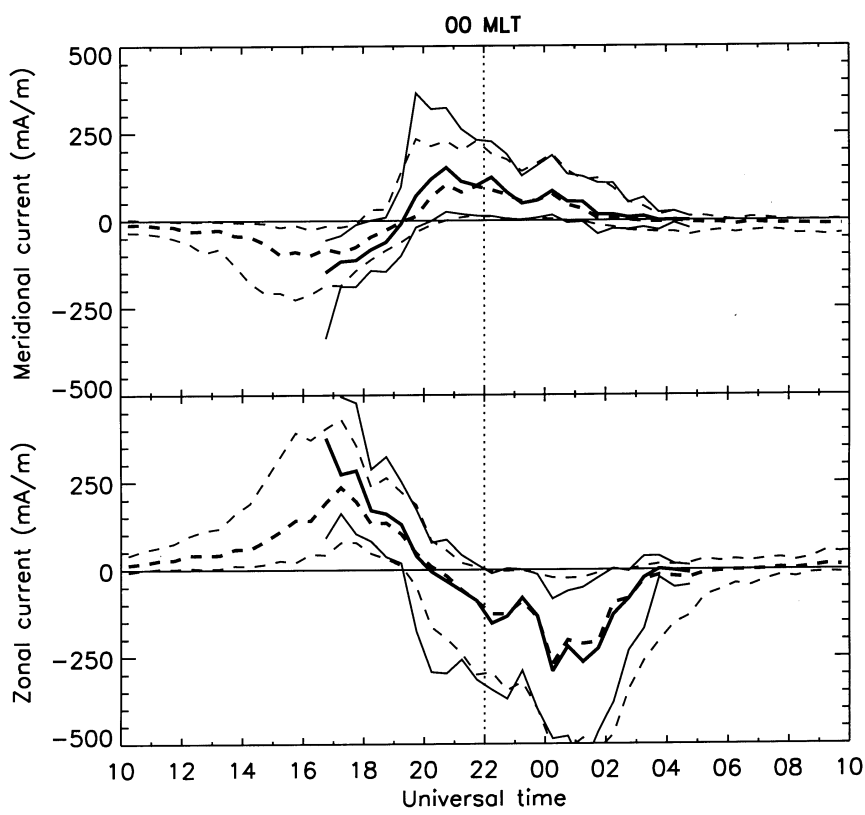

Fig. 3. UT variation of the field-perpendicular north-south current density (upper panel; positive northwards) and the field-perpendicular east-west current density (middle panel; positive eastwards). The format is identical to that of Fig. 1 
conductance rather than that in electric field. The relative contribution to the westward electrojet from enhancements in the conductance and enhancements in the electric field appear to vary considerably, but it should be remembered that the patterns presented are statistical and they vary enormously for individual events, dependant on, for example, substorm phase. This observation of an eastward electrojet controlled by the northward electric field and a westward electrojet dominated, at least in the midnight sector, principally by the Hall conductance, supports on a statistical level the work of, for example, Brekke et al. (1974) and Kamide and Vickrey (1983). Although Kamide and Vickrey (1983) employed an extended data base of observations by the Chatanika incoherent scatter radar, comprising eight sets of data from continuous runs of more than $12 \mathrm{~h}$, this comprises a far smaller data set than that used in the present study. Furthermore, Chatanika, being a monostatic system, does not provide direct measurements of both electric field components. Instead, the authors employed a technique that relies on the rotation of the ion vector velocity with ion-neutral collisions at E-region altitudes to gain estimates of the north-south electric field. The advantage of using the tristatic EISCAT facility in studies of this type is clear, although it should be noted that in using the E-region plasma drift to evaluate the electric field Kamide and Vickrey (1983) do, at least partially, account for currents induced by neutral winds.

It is clear, though, from the present observations that with the transition from eastward to westward directed currents comes a transition from electric field to conductivity driven currents. The second transition, within the body of the westward electrojet itself, from conductivity to electric field dominated currents identified by Kamide and Vickrey (1983), would be expected to be less clear in the present observations. The radar would probably be below the electrojet centre thus moving this transition to later morning hours and possibly out of the regime of the figure.

Previous figures identify the tendency for high conductances to occur in conjunction with low electric fields and vice versa, implying that they are not independent. This relationship may be studied in more detail using simultaneous measurements of conductance and electric field. Figure 4 presents the variation of the Hall conductance (upper panel), Pedersen conductance (middle panel) and the ratio of the Hall and Pedersen conductances (lower panel) with electric field. Solid lines indicate the median and upper and lower quartiles of the conductivities and their ratio, binned with respect to the perpendicular electric field magnitude with a bin width of $5 \mathrm{mV} \mathrm{m}^{-1}$ for fields less than $30 \mathrm{mV} \mathrm{m}^{-1}, 10 \mathrm{mV} \mathrm{m}^{-1}$ for fields between 30 and $60 \mathrm{mV} \mathrm{m}^{-1}$ and $20 \mathrm{mV} \mathrm{m}^{-1}$ for fields exceeding $60 \mathrm{mV} \mathrm{m}^{-1}$. The figures include only observations taken under conditions of solar zenith angle exceeding $105^{\circ}$. For electric fields below some $20 \mathrm{mV} \mathrm{m}^{-1}$, increases in the electric field are accompanied by increases in both Hall and Pedersen conductances; above this limit the conductances decrease as the electric field increases. These features also appear true,



Fig. 4. Variation of the Hall conductance (upper panel), Pedersen conductance (middle panel) and the ratio of the Hall and Pedersen conductances (lower panel) with electric field magnitude. Solid lines indicate the median and upper and lower quartiles of the conductivities and their ratio, binned with respect to the electric field with a bin width of $5 \mathrm{mV} \mathrm{m}^{-1}$ for fields less than $30 \mathrm{mV} \mathrm{m}^{-1}, 10 \mathrm{mV} \mathrm{m}^{-1}$ for fields between 30 and $60 \mathrm{mV} \mathrm{m}^{-1}$ and $20 \mathrm{mV} \mathrm{m}^{-1}$ for fields exceeding $60 \mathrm{mV} \mathrm{m} \mathrm{m}^{-1}$. The figure includes only observations taken under conditions of solar zenith angle exceeding $105^{\circ}$

to some extent, with regard to the ratio between the Hall and Pedersen conductances. Robinson (1984) attributes the anticorrelation between Pedersen conductance and electric field, which the author observed with the Chatanika radar, to a limit on the Pedersen current, itself arising from a limit on field-aligned currents driven by the magnetospheric generator. In the presence of additional field-aligned current systems, however, such as those associated with substorm activity, Robinson (1984) pointed out that the relationship between the Pedersen conductance and the electric field would not be as straightforward.

At lower values of the electric field, those below $20 \mathrm{mV} \mathrm{m}^{-1}$, the correlation between conductances and electric field imply that the EISCAT radar is probably at sub-auroral latitudes.

\section{Summary and conclusion}

The complex interrelationship between electric fields, height-integrated conductivity and electric currents in 
the nightside electrojet region is studied, on a statistical basis, using more than $1300 \mathrm{~h}$ of common programme observations by the EISCAT UHF incoherent scatter radar, taken between 1992 and 1997. Although the electrojet currents can be intensified through enhancements in the conductivity or in the electric field, or indeed a combination of both, the electric field and conductances are not independent; statistical results confirm that, at least in the nightside auroral region for moderate and large electric fields, an increase in the electric field is, in general, accompanied by a decrease in both the Hall and Pedersen conductances and, indeed, their ratio. Consistent with this result is the observation that the times of peak conductivity do not correspond to the times at which the electric field maximises. The dominant factor in current generation in the auroral electrojet region depends critically on local time. Having investigated the statistical properties of conductance and electric field, it is now important to examine these parameters as a function of substorm phase which can be done using magnetometer data, This will form the basis of a follow up study.

Acknowledgements. The authors are indebted to the director and staff of EISCAT for operating the facility and supplying the data. EISCAT is an international association supported by the research councils of Finland (SA), France (CNRS), the Federal Republic of Germany (MPG), Japan (NIPR), Norway (NAVF), Sweden (NFR) and the United Kingdom (PPARC). J. A. Davies is supported by a grant from the Particle Physics and Astronomy Research Council.

Topical Editor D. Alcaydé thanks R. M. Robinson and J. Lilensten for their help in evaluating this paper.

\section{References}

Ahn B. -H., R. M. Robinson, Y. Kamide, and S. -I. Akasofu, Electric conductivities, electric fields and auroral partical energy injection rate in the auroral ionosphere and their empirical relationship to the horizontal magnetic disturbance, Planet. Space Sci., 31, 641-653, 1983.

Aikio A. T., and K. U. Kaila, A substorm observed by EISCAT and other ground-based instruments - evidence for near Earth substorm initiation, J. Atmos. Terr. Phys., 58, 5-21, 1996.

Baumjohann W., Ionospheric and field-aligned current systems in the auroral zone: a concise review, Adv. Space Res., 2, 55-58, 1983.

Brekke A., and C. Hall, Auroral ionospheric quiet time summer conductances. Ann. Geophysicae, 6, 361-376, 1988.

Brekke A., and J. Moen, Review paper: observations of highlatitude ionospheric conductances, J. Atmos. Terr. Phys., 55, 1493-1512, 1993.

Brekke A., R. J. Doupnik, and P. M. Banks, Incoherent scatter measurements of E-region conductivities and currents in the auroral zone, J. Geophys. Res., 79, 3773-3790, 1974.

Brekke A., C. Hall, and T. L. Hansen, Auroral ionospheric conductances during disturbed conditions, Ann. Geophysicae, 7, 269-280, 1989.

Brekke A., C. Hall and Ø. Pettersen, EISCAT UHF studies of ionospheric currents on June 16-17, 1987, Ann. Geophysicae, 8, 213-222, 1990.

Burnside R. G., C. A. Tepley, and V. B. Wickwar, The $\mathrm{O}^{+}-\mathrm{O}$ collision cross-section: can it be inferred from aeronomical measurements?, Ann. Geophysicae, 5, 343-350, 1987.

Craven J. D., Y. Kamide, L. A. Frank, S. -I. Akasofu, and M. Sugiura, Distribution of aurora and ionospheric currents observed simultaneously on a global scale, in Magnetospheric currents, Geophys. Monogr. Ser., 28, Ed. T. A. Potemra, AGU, Washington D. C., 1984.

Davies J. A., M. Lester, B. Jenkins, and R. J. Moffett, Dayside ion frictional heating: EISCAT observations and comparison with model results, J. Atmos. Terr. Phys., 57, 775-793, 1995.

Davis C. J., A. D. Farmer, and A. Aruliah, An optimised method for calculating the $\mathrm{O}^{+}-\mathrm{O}$ collision parameter from aeronomical measurements, Ann. Geophysicae, 13, 541-550-222, 1995.

Foster J. C., J. M. Holt, R. G. Musgrove, and D. S. Evans, Ionospheric convection associated with discrete levels of particle precipitation, Geophys. Res. Lett., 13, 656-659, 1986.

Fuller-Rowell T. J., and D. S. Evans, Height-integrated Pedersen and Hall conductivity patterns inferred from the TIROS-NOAA satellite data, J. Geophys. Res., 92, 76067618, 1987.

Hardy D. A., M. S. Gussenhoven, and E. Holeman, A statistical model of auroral electron precipitation, J. Geophys. Res., 90, 4229-4248, 1985.

Hardy D. A., M. S. Gussenhoven, R. Raistrick, and W. J. McNeil, Statistical and functional representations of the pattern of auroral energy flux, number flux and conductivity, J. Geophys. Res., 92, 12275-12294, 1987.

Horwitz J. L., J. R. Doupnik, P. M. Banks, Y. Kamide, and S. I. Akasofu, The latitudinal distributions of auroral zone electric fields and ground magnetic perturbations and their response to variations in the interplanetary magnetic field, J. Geophys. Res., 83, 2071-2084, 1978.

Kamide Y., and S. Kokubun, Two-component auroral electrojet: importance for substorm studies, J. Geophys. Res., 87, 1302713046, 1996.

Kamide Y., and R. Nakamura, The convection electrojet and the substorm electrojet, Ann. Geophysicae, 14, 589-592, 1996.

Kamide Y., and A. D. Richmond, Ionospheric conductivity dependence of electric fields and currents estimated from ground magnetic observations, J. Geophys. Res., 87, 8331-8337, 1982.

Kamide Y., and J. F. Vickrey, Relative contribution of ionospheric conductivity and electric field to the auroral ionosphere, J. Geophys. Res., 88, 7989-7996, 1983.

Kirkwood S., H. Opgenoorth, and J. S. Murphee, Ionospheric conductivities, electric fields and currents associated with auroral substorms measured by the EISCAT radar, Planet. Space Sci., 36, 1359-1380, 1988.

Lester M., J. A. Davies, and T. S. Virdi, High-latitude Hall and Pedersen conductances during substorm activity in the SUNDIAL-ATLAS campaign, J. Geophys. Res., 101, 26 719-26 728, 1996.

Lilensten J., P. -L. Blelly, W. Kofman, and D. Alcaydé, Auroral ionospheric conductivities: a comparison between experiment and modelling, and theoretical $\mathrm{f}_{10.7}$-dependant model for EISCAT and ESR, Ann. Geophysicae, 14, 1297-1304, 1996.

Moen J., and A. Brekke, The solar flux influence on quiet time conductances in the auroral ionosphere, Geophys. Res. Lett., 20, 971-974, 1993.

Pesnell W. D., K. Omidvar, and W. R. Hoegy, Momentum-transfer collision frequency of $\mathrm{O}^{+}-\mathrm{O}$, Geophys. Res. Lett., 20, 13431346, 1993.

Robinson R. M., $K_{p}$ dependence of auroral zone field-aligned current intensity, J. Geophys. Res., 89, 1743-1748, 1984.

Robinson R. M., R. R. Vondrak, K. Miller, T. Dabbs, and D. Hardy, On calculating ionospheric conductances from the flux and energy of precipitating particles, J. Geophys. Res., 92, 25652569, 1987.

Salah J. E., Interim standard for the ion-neutral atomic oxygen collision frequency, Geophys. Res. Lett., 20, 1543-1546, 1993.

Schlegel K., Auroral zone E-region conductivities during solar minimum derived from EISCAT data, Ann. Geophysicae, 6, 129-138, 1988.

Schunk R. W., and A. F. Nagy, Electron temperatures in the Fregion of the ionosphere: theory and observations, Rev. Geophys. Space Phys., 16, 355-399, 1978. 
Schunk R. W., and A. F. Nagy, Ionospheres of terrestrial planets, Rev. Geophys. Space Phys., 18, 813-852, 1980.

Senior C., Solar and particle contributions to auroral heightintegrated conductivities from EISCAT data: a statistical study, Ann. Geophysicae, 9, 449-460, 1991.

Senior C., R. M. Robinson, and T. A. Poterma, Relationship between field-aligned currents, diffuse auroral precipitation and the westward electrojet in the early morning sector, J. Geophys. Res., 87, 10 469-10 477, 1982.
Spiro R. W., P. H. Reiff, and L. J. Maher Jr., Precipitating electron energy flux and auroral zone conductances - an empirical model, J. Geophys. Res., 87, 8215-8227, 1982.

Vickrey J. F., R. R. Vondrak, and S. J. Matthews, The diurnal and latitudinal variation of auroral zone ionospheric conductivity, J. Geophys. Res., 86, 65-75, 1981. 